\title{
Incidence of macrovascular disease in diabetes mellitus: the London cohort of the WHO Multinational Study of Vascular Disease in Diabetics
}

\author{
N.J.Morrish ${ }^{1}$, L.K.Stevens ${ }^{3}$, J.H. Fuller ${ }^{3}$, H.Keen ${ }^{1}$ and R.J. Jarrett ${ }^{2}$ \\ Unit for Metabolic Medicine and ${ }^{2}$ Department of Community Medicine, United Medical and Dental Schools (Guy's Campus) and \\ ${ }^{3}$ Department of Community Medicine, University College, London, UK
}

Summary. We report on the incidence of new macrovascular disease among the 497 members of the London Cohort of the WHO Multinational Study of Vascular Disease in Diabetics (aged 35-54 years at recruitment) over a mean 8.33 year follow-up period. Overall at the end of the follow-up period the prevalence of macrovascular disease in the cohort was $45 \%$; $43 \%$ of the subjects showed evidence of ischaemic heart disease, $4.5 \%$ of cerebrovascular disease and $4.2 \%$ of peripheral vascular disease. The incidence rates for new disease in those subjects who were free at baseline expressed per 1000 patient years of follow-up were: ischaemic ECG abnormality 23.6 (patients with insulin-dependent diabetes 19.8, patients with non-insulin-dependent diabetes 28.1), myocardial infarction 17.6 (patients with insulin-dependent diabetes 16.5 , patients with non-insulin-dependent diabetes 18.8 ), all ichaemic heart disease 31.7 (patients with insulin-dependent diabetes 30.3, patients with non-insulin-dependent diabetes 33.4 ), cerebrovascular disease 5.9 and peripheral vascular disease 5.2. Incidence rates were generally similar among men and women except for myocardial infarction in patients with non-insulindependent diabetes where men had a significantly higher incidence rate. Macrovascular disease is a major problem in patients with diabetes and in this age group is mainly manifested as ischaemic heart disease.

Key words: Insulin-dependent diabetes mellitus, non-insulin-dependent diabetes mellitus, ischaemic heart disease, myocardial infarction, electrocardiography, cerebrovascular disease, peripheral vascular disease, incidence.
Much of the ill health and premature mortality in patients with diabetes mellitus is caused by macrovascular disease; ischaemic heart disease, peripheral vascular disease or cerebrovascular disease either alone or in combination.

The WHO Multinational Study of Vascular Disease in Diabetics has investigated international differences in the prevalence of the vascular complications of diabetes [1] and associated mortality rates [2,3]. Patients recruited for the Multinational Study were between 35 and 54 years old at entry: an age range chosen to be likely to display the emergence of vascular complications but not yet to be unduly distorted by premature mortality. We report here the incidence of new macrovascular events in the 497 patients of the London cohort over a mean 8.33 year follow-up.

\section{Subjects and methods}

Recruitment for the WHO Multinational Study was based on attained age and diabetes duration: men and women with diabetes were divided into one of three age groups (35-41 years, 42-
48 years, 49-55 years) and one of three groups based on diabetes duration ( $>6$ years, $7-14$ years, $>14$ years): selection for each of nine age/duration cells was then made randomly and separately for each sex. The patients were recruited from King's College, Guy's and St. Thomas's Hospitals in London. A diabetic patient was defined as a person who had been receiving treatment for diabetes at one of the participating hospitals for more than a year. In this report an insulin-dependent patient is defined as one who started insulin treatment within one year of diagnosis and who had continued to use it subsequently and continuously. All others are regarded as having non-insulin-dependent diabetes. Of the 497 patients entering the study in London 254 were men and 245 were women. The composition of the London Cohort by sex and diabetes type is shown in Table 1.

At baseline each patient was submitted to a standard examination. This included completion of the WHO questionnaire for symptoms of cardiovascular disease [4] and enquiry about diabetes diagnosis, duration and treatment. Full details of the WHO Multinational Study procedures have been published previously [1]. Height, weight and arterial blood pressure were measured, urine was tested for proteinuria using the salicylsulphonic acid method [5] and venous blood was taken for the estimation of total cholesterol and creatinine. A resting 12-lead electrocardiogram (ECG) was recorded and Minnesota coded [4] independently by two experienced observers; these observers coded both the baseline and follow-up electrocardiograms. 
Table 1. The London cohort of the WHO Multinational Study of Vascular Disease in Diabetics at baseline by sex and diabetes type

\begin{tabular}{lll}
\hline & Men & Women \\
\hline Patients with insulin-dependent diabetes & 118 & 125 \\
Patients with non-insulin-dependent diabetes & 136 & 118 \\
\hline
\end{tabular}

(Diabetes type defined by interval between diagnosis and starting continuous insulin treatment: $<1$ year = insulin-dependent diabetes, other $=$ non-insulin-dependent diabetes)

Those who died during the follow-up period were traced via the National Health Service Central Registry who provided a copy of the death certificate. Attempts were then made to obtain as much further information as possible from clinical records and/or autopsy results. On the basis of all available information an underlying cause of death was agreed for each patient by a committee of four physicians. This agreed cause is used in the analysis.

The surviving patients were invited to attend a follow-up examination with almost the same format as the baseline ascertainment. Home visits were offered to patients not attending for re-examination; if this was not possible then systematic enquiries were made to get information from the patient, from their general practitioner and from any hospital clinic they were attending. The follow-up status of the cohort is shown in Table 2 . The information collected during the 33 home visits was identical to that obtained in those patients who attended the hospitals for follow-up and included an electrocardiogram. In general the reason for performing a home visit was because the patients were unwilling to come for a special study visit rather than because of incapacity due to illness.

The baseline and follow-up information has been used to create outcome variables for macrovascular complications. For each complication the patients are divided into four groups 1) those who had evidence of a complication at baseline, 2) those who developed that complication during follow-up, 3) those without evidence of that complication at the end of the follow-up and 4) those for whom there is insufficient or no follow-up information regarding the complication.

The status at follow-up is defined by outcome variables including cause of death (where appropriate), results of the follow-up questionnaire and investigations, and patient and physician reported information. Outcome variables for 'ischaemic ECG abnormality', 'myocardial infarction', 'ischaemic heart disease', 'peripheral vascular disease' and 'cerebrovascular disease' have been defined. Angina pectoris has not been analysed separately as an outcome but is used in the definition of ischaemic heart disease. Ischaemic heart disease includes those subjects with evidence of any of the following: myocardial infarction, angina pectoris or ischaemic ECG abnormality (ischaemic heart disease possible or probable [below]). Myocardial infarction, ischaemic heart disease and cerebrovascular disease include both fatal and non-fatal events: peripheral vascular disease incidence relates only to surviving patients.

The outcomes were defined as follows:

\section{Ischaemic ECG abnormality}

This employed the same definitions in both original and follow-up phases using Minnesota coding to identify three groups 1 ) ischaemic heart disease probable; major and medium $\mathrm{Q}$ waves or complete left bundle branch block (Minnesota codes 1-1, 1-2, 7-1), 2) ischaemic heart disease possible; ST segment abnormalities if accompanied by abnormal $T$ waves, minor Q/QS wave changes (Minnesota codes $1-3$, or any of $4-1,4-2,4-3$ if accompanied by $5-1$ or $5-2$ or 5-3, 3) ischaemic heart disease unlikely (all other Minnesota codes). ECG results in the analyses in this report are classified as abnormal; groups 1) and 2) above, or normal; group 3). Only those patients in whom a second ECG was obtained could be included in the ECG follow-up results and the numbers ascertained for this outcome are therefore considerably smaller than for any of the other macrovascular outcomes.

\section{Myocardial infarction}

a) At baseline: A patient was considered to have had a myocardial infarction if either their ECG showed 'definite ischaemic heart disease' or they answered 'yes' to the question on 'severe chest pain lasting 30 min or more' in the WHO Cardiovascular Questionnaire ('pain of possible infarction') [4] or there was evidence in the followup questionnaires of a medically authenticated and dated myocardial infarction occurring before the date of the original interview.

b) Atfollow-up: The definition for a myocardial infarction at followup is similar to that at baseline using the ECG changes and the "pain of possible infarction' question in the WHO Cardiovascular Questionnaire [4]. In addition those cases with myocardial infarction as the underlying cause of death or those with a medically authenticated and dated myocardial infarction during the follow-up were included as positive.

\section{Angina pectoris}

Angina at baseline and follow-up was defined by positive answers to the WHO Cardiovascular Questionnaire [4]. The follow-up definition for angina also included new angina during the study period in patients not fully re-ascertained if medically authenticated and dated.

\section{Total ischaemic heart disease}

Patients were defined as having ischaemic heart disease at baseline if they had either an abnormal ECG (possible or definite ischaemic heart disease) or angina or evidence of myocardial infarction (as defined above). Ischaemic heart disease at follow-up was defined using the same criteria with the addition of deaths with ischaemic heart disease as the underlying cause of death.

\section{Cerebrovascular disease}

Cerebrovascular disease at baseline was defined by the response to the question on stroke in the original questionnaire. This was repeated in the main follow-up questionnaire. The follow-up definition for cerebrovascular disease also included new cerebrovascular events during the study period in patients not fully re-ascertained if medically authenticated and dated.

\section{Peripheral vascular disease}

This was defined at baseline by appropriately positive answers to the questions about leg pain in the WHO Cardiovascular Questionnaire [4]. At follow-up, in addition to this, patients with new medically

Table 2. The follow-up status of the cohort

\begin{tabular}{|c|c|c|c|c|}
\hline & \multicolumn{2}{|c|}{$\begin{array}{l}\text { Patients with } \\
\text { insulin-dependent } \\
\text { diabetes }\end{array}$} & \multicolumn{2}{|c|}{$\begin{array}{l}\text { Patients with non- } \\
\text { insulin-dependent } \\
\text { diabetes }\end{array}$} \\
\hline & $n$ & $\%$ & $n$ & $\%$ \\
\hline Died before follow-up & 20 & 8.2 & 34 & 13.4 \\
\hline Seen at follow-up & 189 & 77.8 & 166 & 65.3 \\
\hline $\begin{array}{l}\text { Questionnaire information } \\
\text { only }\end{array}$ & 26 & 10.7 & 35 & 13.8 \\
\hline $\begin{array}{l}\text { Alive but no other infor- } \\
\text { mation }\end{array}$ & 5 & 2.1 & 11 & 4.3 \\
\hline Life/death unknown & 3 & 1.2 & 8 & 3.2 \\
\hline
\end{tabular}




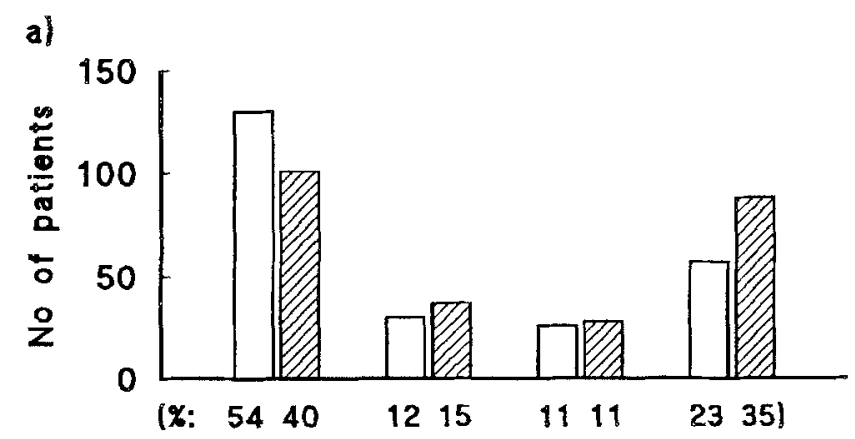

b)

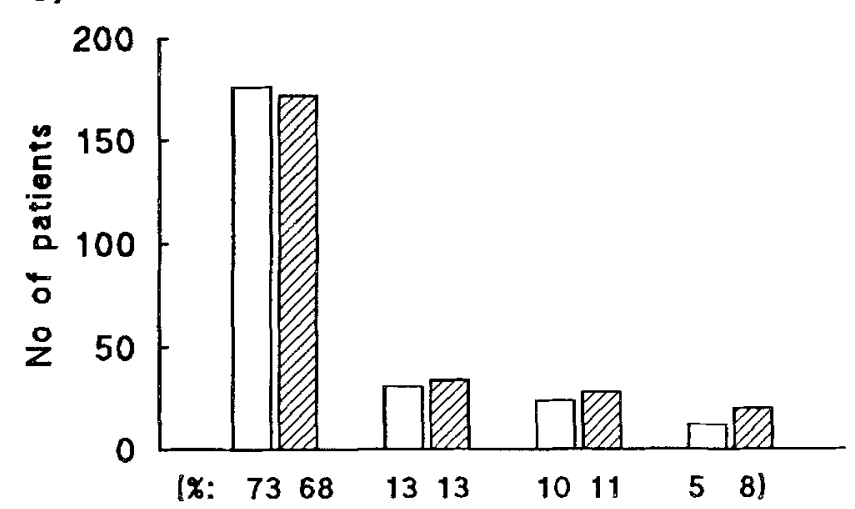

c)

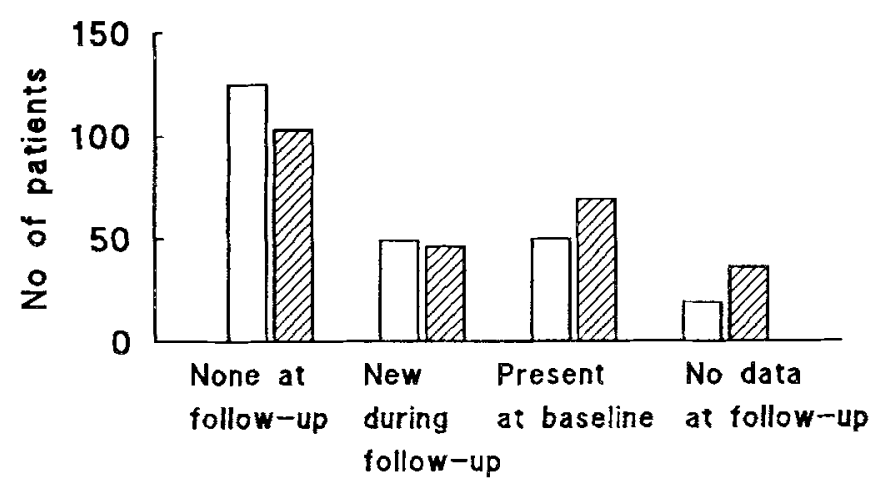

(\%: $\begin{array}{llllllll}51 & 41 & 20 & 18 & 21 & 27 & 8 & 14\end{array}$

Fig. 1a-c. Numbers of patients categorised with regard to ischaemic heart disease outcome variables at the end of the follow-up period. $(\square$ = Patients with insulin-dependent diabetes, $\square=$ Patients with non-insulin-dependent diabetes). a ischaemic ECG abnormality, b myocardial infarction, $\mathbf{c}$ all ischaemic heart disease. The numbers below each panel are percentages for each diabetes type in the bar above

authenticated and dated peripheral vascular disease during the follow-up period were also included. Amputations were only included if they were due predominantly to arterial disease, amputations for neuropathic disease being excluded. No information about peripheral vascular disease in the non-survivors is included as no consistent method of ascertainment could be devised. No patient died from causes related to peripheral vascular disease.

\section{Results}

Figure 1 shows the numbers of patients with either type of diabetes in each of the categories for ischaemic ECG abnormality, myocardial infarction and total ischaemic

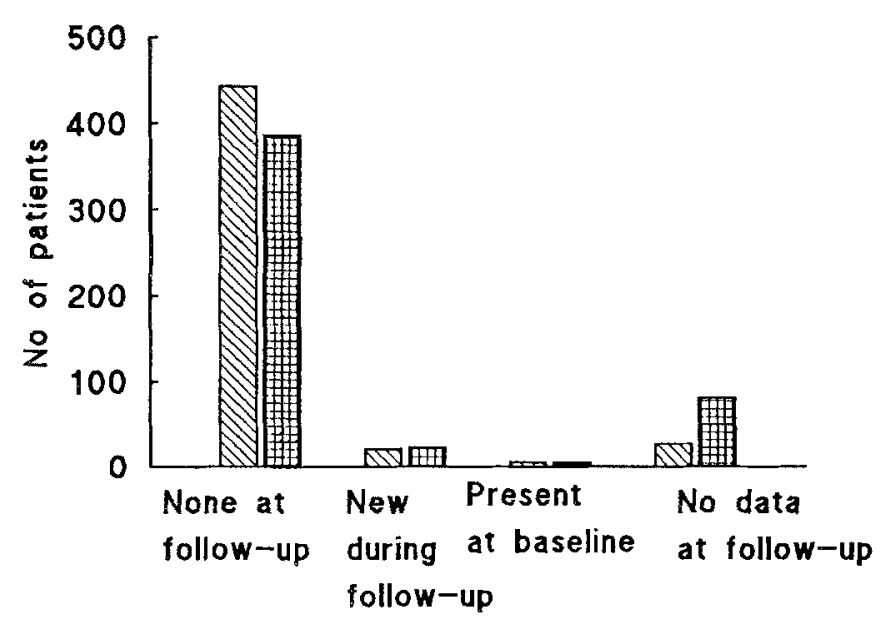

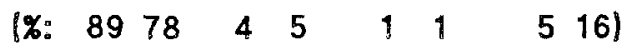

Fig. 2. Numbers of patients categorised with regard to cerebrovascular and peripheral vascular disease outcome at the end of the followup. $(\mathrm{S})=$ cerebrovascular disease, = peripheral vascular disease). The numbers below refer to percentages of the cohort in the bar above

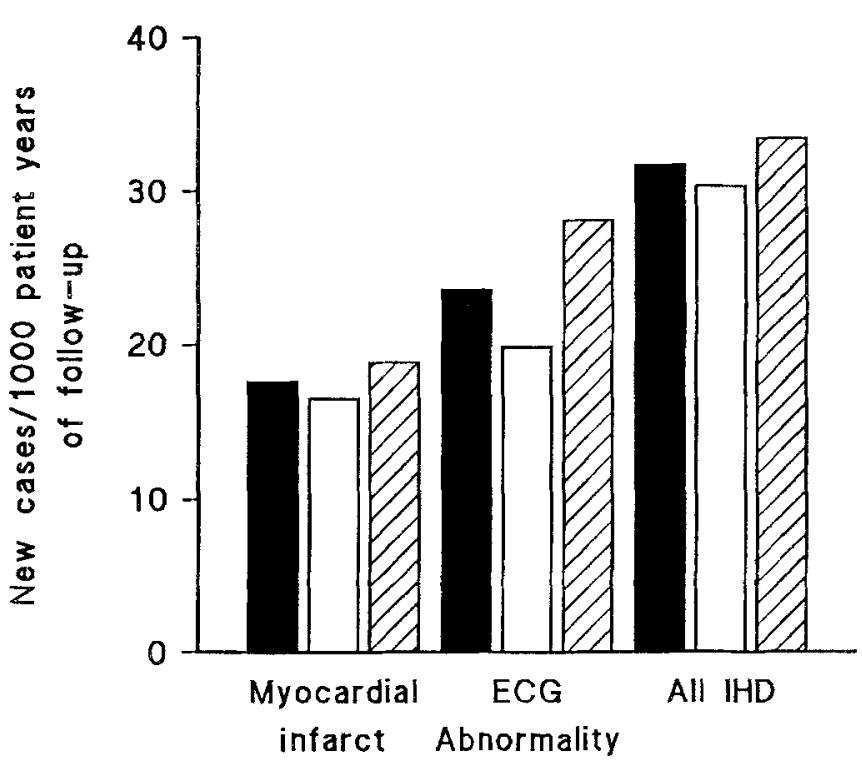

Fig.3. Incidence rates for new ischaemic ECG abnormality, new myocardial infarction and all new ischaemic heart disease (IMD) during the follow-up period. ( $\boldsymbol{D}=$ All patients, $\square=$ Patients with insulin-dependent diabetes. dent diabctes)

heart disease at the end of the follow-up. Results for peripheral vascular disease and cerebrovascular disease are shown in Figure 2, these results are presented for the whole group as the numbers of incident events were small. Variation in the number of patients without followup information is due to the way that the various categories are defined.

At the end of a mean 8.33 year follow-up (range 6 10.1 years) $45 \%$ of the cohort (224 patients) showed evidence of some manifestation of macrovascular disease, $43 \%$ (214 patients) appeared free of all forms of macrovascular disease, with insufficient evidence to make a diagnosis in the remaining 59 patients. There was evidence of is- 
a)

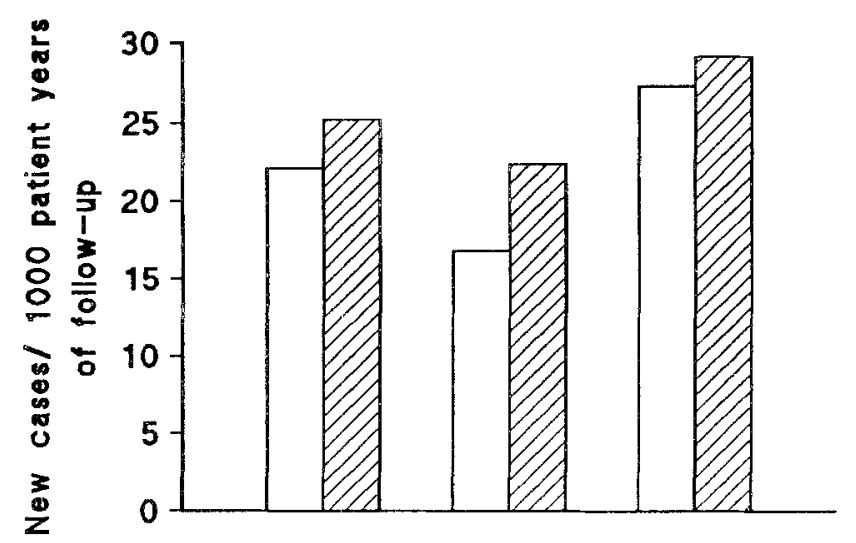

b)

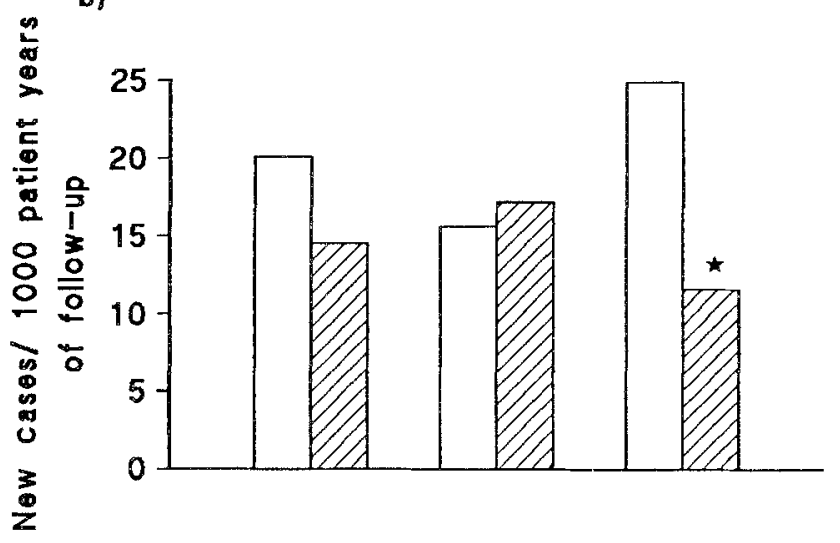

c)

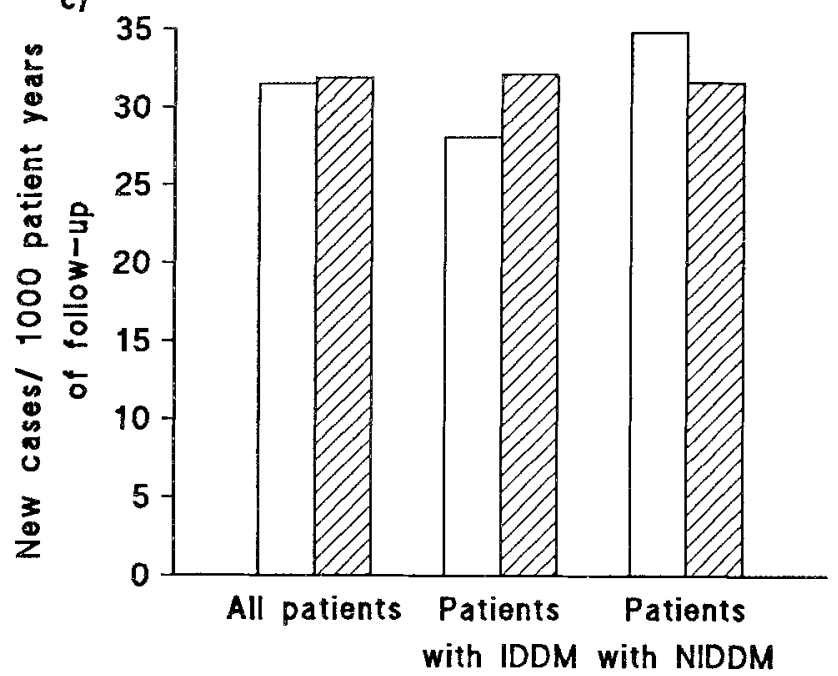

Fig. 4 a-c. Incidence rates for a new ischaemic ECG abnormality, $b$ new myocardial infarction and $\mathbf{c}$ all new ischaemic heart disease during the follow-up by sex. $(\square=$ men, $\nabla \lambda=$ women $) * p<0.05$

chaemic heart disease in $43 \%$ of the original 497 patients after the follow-up; 161 out of 387 living patients and 53 out of the 92 who had died. $23.6 \%$ showed evidence of myocardial infarction and $24.3 \%$ had an abnormal ECG. The proportions for cerebrovascular disease $(4.5 \%)$ and peripheral vascular disease $(4.2 \%)$ are much lower.

Figure 3 shows the incidence rates for the ischaemic heart disease outcome variables in those subjects free of disease at baseline expressed as new cases per 1000 pa- tient years of follow-up. There were too few cases of cerebrovascular disease and peripheral vascular disease to calculate separate rates for the two diabetes types. For the group as a whole the rates are: cerebrovascular disease; 5.9 new cases per 1000 patient years and for peripheral vascular disease; 5.1 new cases per 1000 patient years. The rates for ischaemic heart disease events are about 6 times higher than those for peripheral vascular disease and cerebrovascular disease in this age group. The rates for total ischaemic heart disease and for myocardial infarction are similar for patients with either type of diabetes. Although new ischaemic ECG abnormalities appear more common in patients with non-insulin-dependent than in those with insulin-dependent diabetes, the $95 \%$ confidence intervals for the rate difference are wide and the difference is nonsignificant.

Figure 4 shows the incidence rates for new ischaemic heart disease events calculated by sex for the whole cohort and for either diabetes type. Whilst the rates for new total ischaemic heart disease and for new ischaemic ECG change are similar for men and women there is a significantly higher rate of new myocardial infarction amongst men with non-insulin-dependent diabetes than among women with non-insulin-dependent diabetes $(p<0.05$, rate difference [men - women] 13.73 cases per 1000 years of follow-up, 95\% confidence interval 1.5-25.9). The similar overall ischaemic heart disease rate in the two sexes for patients with non-insulin-dependent diabetes is accounted for by a higher incidence rate of new angina in women with non-insulin-dependent diabetes than in men.

\section{Discussion}

Almost half of the cohort had acquired evidence of ischaemic heart disease by the end of the follow-up. $21.4 \%$ of those followed-up developed new ischaemic heart disease during the follow-up, an incidence of 31.7 per 1000 patient years of follow-up. In view of the difficulties in ascertaining ischaemic heart disease in those who died of other causes, the calculated rates are likely to be underestimates of the true incidence. Incidence was similar for the two types of diabetes as was the incidence of all ischaemic heart disease and myocardial infarction expressed per 1000 patient years of follow-up. Incidence per 1000 patient years of new ischaemic ECG abnormality was higher in patients with non-insulin-dependent diabetes than in patients with insulin-dependent diabetes but the rates as crude percentages are similar. There are few comparable studies in the literature but the figures resemble those reported by Krolewski et al. [6] from the Joslin Clinic in an American cohort of patients with insulindependent diabetes where the cumulative incidence of fatal and non-fatal coronary artery disease was $50 \%$ by age 50 years. The average annual incidence of cardiovascular disease per 1000 diabetic patients aged 55-64 years at risk in the Framingham population was 48.1 for men and 37.9 for women; in the age-group 45-54 years rates were 31.7 for men and 24.8 for women [7]. In the London follow-up, overall incidence rates for ischaemic heart disease were very similar in the two sexes; myocardial in- 
farction rates were significantly higher for men than women with non-insulin-dependent diabetes but in patients with insulin-dependent diabetes this was not seen. The finding that rates in men and women are similar in insulin-dependent diabetes is in accord with the results on mortality [3] in this group where we found that the female survival advantage seen in the general population in this age group did not appear to operate in patients with insulin-dependent diabetes. The mortality results suggested that among patients with non-insulin-dependent diabetes there was some female advantage and to a degree this may be reflected here with a significantly higher incidence rate of myocardial infarction among men with non-insulin-dependent diabetes. This sex difference was, however, only found in the incidence rates for myocardial infarction and not in the other two ischaemic heart disease outcomes studied and it is difficult to be sure of its biological significance. Secular changes in cardiovascular disease in both the USA and the UK over the last two decades may contribute to the higher, earlier US rates.

In a clinic population in Birmingham, UK with a mean age of 49 years, Shapiro [8] reported a prevalence of $19 \%$ for Minnesota coded ECG abnormalities suggestive of myocardial ischaemia or infarction which is similar to the prevalence after the follow-up reported here. The Birmingham patients were a mixture of new and established cases and duration was not reported.

There were relatively few cerebrovascular events during the follow-up with $4.4 \%$ ( 21 cases) of those followedup becoming affected during the follow-up; an incidence rate of 5.9 per 1000 patient years of follow-up, about $20 \%$ of the rate for total ischaemic heart disease. A recent Finnish report of patients with non-insulin-dependent diabetes aged 45-64 years found a prevalence of $5.7-8.0 \%$ for men and 3.6-5.1\% for women of 'verified definite or possible stroke' [9]. This is similar to the final prevalence in the whole London cohort. The average annual incidence for 'atherothrombotic brain infarction' in the Framingham diabetic population was $1.0-2.0$ per $1000 \mathrm{pa}-$ tients at risk for men in the 45-64 year age range and $0.6-1.8$ for women, rising to 5.2 for men and 5.5 for women aged $65-75$ years. Overall age-adjusted annual incidence rates for patients with diabetes were 4.7 per 1000 for men and 6.2 per 1000 for women; for the general Framingham population the rates were 1.9 and 1.7 per 1000 respectively [10]. These figures exclude cerebral haemorrhage and embolic cerebral infarction.

The rates for new cases of peripheral vascular disease are also much lower than those for ischaemic heart disease with 21 new cases during the follow-up (5.9 per 1000 patient years of follow-up). Lower extremity amputations are 15 times more common in patients with diabetes than in those without [11]. It is therefore surprising that the rates of peripheral vascular disease as evidenced by symptoms of intermittent claudication and/or gangrene/amputation for arterial disease were not higher in the London WHO follow-up (although they may have been higher than rates in a non-diabetic population). A recent report from the Framingham study gives age-adjusted 2-year incidence rates for intermittent claudication among patients with diabetes of 15.6 per 1000 for men and 9.1 per 1000 for women aged less than 50 years and considerably higher in older age groups [12]. Kreines et al. [13] have reviewed the literature on peripheral vascular disease in patients with non-insulin-dependent diabetes and quote a composite incidence rate of 8-12 per 1000 person years for intermittent claudication in out-patient studies. Using a similar definition of intermittent claudication to that used in the WHO Multinational Study they reported annual incidences for intermittent claudication of 29 per 1000 men and 19 per 1000 women in a population of 619 patients with non-insulin-dependent diabetes followed-up for 13 years but with low rates of amputation; they do not quote the ages of their patients. The Finnish study of Laakso et al. [9] in patients with non-insulin-dependent diabetes of a similar age to our patients also used the WHO questionnaire and found a prevalence of intermittent claudication of $5.4-21.6 \%$ in men (depending on the part of the country) and $5.8-7.7 \%$ in women. The rates in London therefore seem low compared to those from other centres although differing methods of patient selection, ascertainment and analysis make direct comparison between studies difficult. It is to be hoped that the provision of specialised diabetes foot services in recent years may have lowered the rate of amputations in this hospital-based cohort. The true incidence of peripheral vascular disease in this diabetic cohort may have been masked by the loss from the cohort by deaths from ischaemic heart disease with which it is strongly associated $[12,13]$.

This study reaffirms the important problem of macrovascular disease in people with diabetes. In the age group studied the risk from ischaemic heart disease overshadows the impact of the disease of the peripheral and cerebral circulations.

Acknowledgements. The project was supported by a Medical Research Council Project Grant. We would like to thank the staff of the Diabetic Departments on King's College Hospital and St. Thomas' Hospital for their cooperation.

\section{References}

1. The World Health Organisation Multinational Study of Vascular Disease in Diabetics (1985) Prevalence of smail vessel and large vessel disease in diabetic patients from 14 centres. Diabetologia 28 [Suppl.]: 615-640

2. Head J, Fuller JH (1990) International variations in mortality among diabetic patients: The WHO Multinational Study of Vascular Disease in Diabetics. Diabetologia 33: 477-482

3. Morrish NJ, Stevens L, Head JA, Fuller JH, Jarrett RJ, Keen H (1990) A prospective study of mortality among middle-aged diabetics (the London Cohort of the WHO Multinational Study of Vascular Disease in Diabetics) I: Causes and death rates. Diabetologia 33: $538-541$

4. Rose G, Blackburn H (1968) Cardiovascular Survey Methods. WHO Monograph Series No 56, WHO Geneva

5. Rennie IDB, Keen H, Cowhig J, Field M, Harvey M, Quartey E (1967): Evaluation of clinical methods for detecting proteinuria. Lancet II: 489-492

6. Krolewski AS, Kosinski EJ, Warram JH, Leland S, Busick EJ, Asmal AC, Rand LI, Christlieb AR, Bradley RF, Kahn CR (1987) Magnitude and determinants of coronary artery disease in 
juvenile onset, insulin-dependent diabetes mellitus. Am J Cardiol 59:750-755

7. Kannel WB, McGee DL (1979b) Diabetes and cardiovascular risk factors: The Framingham study. Circulation 59:8-13

8. Shapiro LM (1984) A prospective study of heart disease in diabetes mellitus. Quart J Med 209: 55-68

9. Laakso M, Ronnemaa R, Pyorala K, Kallio V, Puukka P, Penttila I (1988) Atherosclerotic vascular disease and its risk factors in non-insulin-dependent diabetic and nondiabetic subjects in Finland. Diab Care 11: 449-463

10. Kannel WB, McGee DL (1979a) Diabetes and cardiovascular disease: the Framingham study. JAMA 241: 2035-2038

11. Most RS, Sinnock P (1983) The epidemiology of lower extremity amputations in diabetic individuals. Diab Care 6:87-91

12. Brand FN, Abbott RD, Kannel WB (1989) Diabetes, intermittent claudication and risk of cardiovascular events. Diabetes 38 : 504-509
13. Kreines K, Johnson E, Albrink M, Knatterud GL, Levin ME, Lewitan A, Newberry W, Rose FA (1985) The course of peripheral vascular disease in non-insulin-dependent diabetes. Diab Care 8: 235-243

Received: 7 January 1990

and in revised form: 17 April 1991

Dr. N.J.Morrish

Unit for Metabolic Medicine

Guy's Hospital

London SE1 9RT

UK 